Proc. 18th Winter Workshop on

Nuclear Dynamics (2002) 000-000

18th Winter Workshop on Nuclear Dynamics

Nassau, Bahamas

January 20-27, 2002

\title{
Probing Hadronization with Strangeness
}

\author{
S.A. Bass ${ }^{1,2}$, M. Bleicher ${ }^{3}$, J. Aichelin ${ }^{3}$, F. Becattini ${ }^{4}$, A. Keränen ${ }^{5}$,

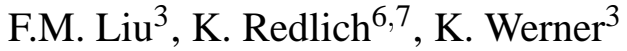 \\ ${ }^{1}$ Department of Physics, Duke University \\ Durham, NC 27708-0305, USA \\ 2 RIKEN-BNL Research Center, Brookhaven National Laboratory \\ Upton, NY 11973, USA \\ ${ }^{3}$ SUBATECH, Laboratoire de Physique Subatomique et des Technologies Associées \\ University of Nantes - IN2P3/CNRS - Ecole des Mines de Nantes \\ 4 rue Alfred Kastler, F-44072 Nantes, Cedex 03, France \\ ${ }^{4}$ Universitá di Firenze and INFN Sezione di Firenze \\ Via G. Sansone 1, I-50019, Sesto F.no, Florence, Italy \\ ${ }^{5}$ Department of Physics, Theoretical Physics \\ 90014 University of Oulu, Finland \\ 6 Theory Division, CERN \\ CH-1211 Geneva 23, Switzerland \\ ${ }^{7}$ Institute for Theoretical Physics, University of Wroclaw \\ PL-50204 Wroclaw, Poland
}

\begin{abstract}
The $\bar{\Omega} / \Omega$ ratio originating from string decays is predicted to be larger than unity in proton-proton interaction at SPS energies. The anti-omega dominance increases with decreasing beam energy. This surprising behavior is caused by the combinatorics of quark-antiquark production in small and low-mass strings. Since this behavior is not found in a statistical description of hadron production in proton-proton collisions, it may serve as a key observable to probe the hadronization mechanism in such collisions.
\end{abstract}

Keywords: hadronization, statistical models, string fragmentation PACS: 25.75.-q 


\section{Introduction}

Hadron yields and their ratios stemming from the final state of ultra-relativistic heavyion collisions have been extensively used to explore the degree of chemical equilibration [ 1, 2, 3, 4, 5, 6, 7, 8, 9, 10, and to search for evidence for exotic states and phase transitions in such collisions [1]. Under the assumption of thermal and chemical equilibrium, fits with a statistical (thermal) model have been used to extract bulk properties of hot and dense matter, e.g. the temperature and chemical potential at which chemical freeze-out occurs [ 5, 6, 7, 8, 9.

The application of a statistical model to elementary hadron-hadron reactions was first proposed by Hagedorn [ 11] in order to describe the exponential shape of the $m_{t}$-spectra of produced particles in $\mathrm{p}+\mathrm{p}$ collisions. Recent analyses [ 12] on hadron yields in electronpositron and proton-proton interactions at several centre-of-mass energies have shown that particle abundances as well can be described by a statistical ensemble with maximized entropy. In fact, the abundancies are consistent with a model assuming the existence of equilibrated fireballs at a temperature $T \approx 160-170 \mathrm{MeV}$. These findings have given renewed rise to the interpretation that hadronization in elementary hadron-hadron collisions is a purely statistical process, which is difficult to reconcile with the popular dynamical picture that hadron production in pp collisions is due to the decay of color flux tubes [13].

In this article we argue that the $\bar{\Omega} / \Omega \equiv \Omega^{+} / \Omega^{-}$ratio in elementary proton-proton collisions is an unambiguous and sensitive probe to distinguish between particle production via the breakup of a color flux tube from statistical hadronization [14].

\section{2. (Anti-)baryon production in sting models}

Color flux tubes, called strings, connect two SU(3) color charges [ 3 ] and [ $\overline{3}$ ] with a linear confining potential. If the excitation energy of the string is high enough it is allowed to decay via the Schwinger mechanism [ 15], i.e. the rate of newly produced quarks is given by:

$$
\frac{\mathrm{d} N_{\kappa}}{\mathrm{d} p_{\perp}} \sim \exp \left[-\pi m_{\perp}^{2} / \kappa\right]
$$

where $\kappa$ is the string tension and $m_{\perp}=\sqrt{p_{\perp}^{2}+m^{2}}$ is the transverse mass of the produced quark with mass $m$.

However, specific string models may differ in their philosophy and the types of strings that are created:

- In UrQMD[ 16] the projectile and target protons become excited objects due to the momentum transfer in the interaction. The resulting strings, with at most two strings being formed, are of diquark-quark type.

- In NeXuS[ 17], the pp interaction is described in terms of pomeron exchanges or ladder diagramms. Both hard and soft interactions happen in parallel. Energy is shared equally between all cut pomerons and the remnants. The endpoints of the 
cut pomerons (i.e. the endpoints of the strings) may be valence quarks, sea quarks, antiquarks or gluons.

- In PYTHIA[ 18], a similar scheme as in UrQMD is employed. However, hard interactions may create additional strings from scattered sea quarks. Most strings are also of diquark-quark form.

Fig.17a depicts the anti-baryon to baryon ratio at midrapidity in proton-proton interactions at $160 \mathrm{GeV}$. The results of the calculations by NeXuS, UrQMD and PYTHIA, which are the best established string-fragmentation models for elementary hadron-hadron interactions, are included in this figure. In all these models, the $\bar{B} / B$ ratio increases strongly with the strangeness content of the baryon. For strangeness $|s|=3$ the ratio significantly exceeds unity. In UrQMD and PYTHIA the hadronization of the diquark-quark strings leads directly to the overpopulation of $\bar{\Omega}$. In NeXuS however, the imbalance of quarks and anti-quarks in the initial state leads to the formation of $q_{\mathrm{val}}-\bar{s}_{\text {sea }}$ strings, (the $s_{\mathrm{val}}-\bar{q}_{\text {sea }}$ string is not possible). These strings than result in the overpopulation of $\bar{\Omega}$.

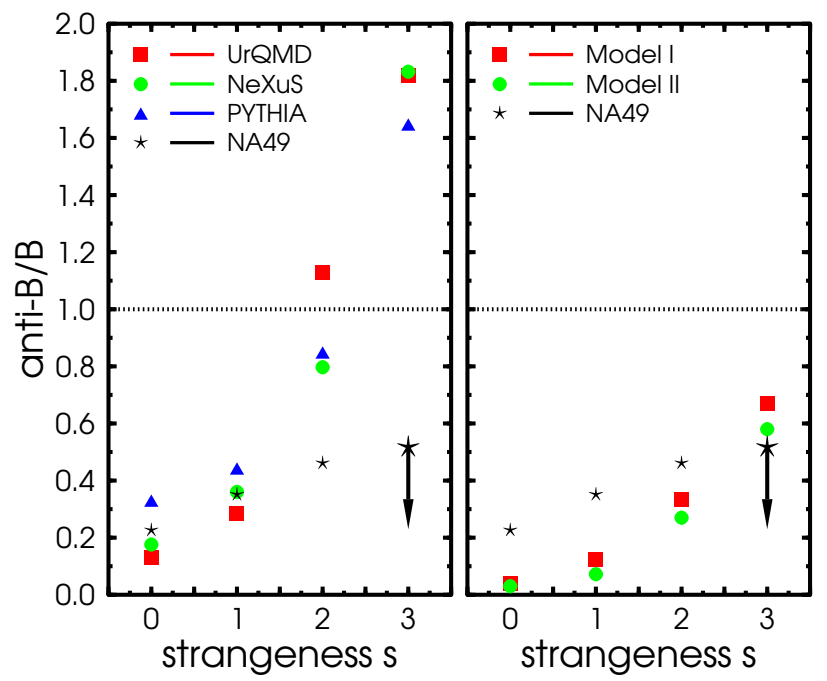

Fig. 1. Left: anti-baryon to baryon ratio at midrapidity in pp interactions at $160 \mathrm{GeV}$ as given by PYTHIA, NeXuS and UrQMD as well as statistical model. Right: anti-baryon to baryon ratio in $4 \pi$ for the same reaction as given by statistical models. Stars depict preliminary NA49 data for the $\bar{B} / B$ ratio at midrapidity.

\section{3. (Anti-)baryon production in statistical models}

In Fig. 1] the string model results are compared with the predictions of statistical models (SM). Within the SM, hadron productions is commonly described using the grand canonical (GC) partition function, where the charge conservation is controlled by the related 
chemical potential. In this description a net value of a given $\mathrm{U}(1)$ charge is conserved on average. However, in the limit of small particle multiplicities, conservation laws must be implemented exactly, i.e., the canonical (C) ensemble for conservation laws must be used [12, 19, 20]. The conservation of quantum numbers in the canonical approach severely reduces thermal phase space available for particle production. Thus, exact charge conservation is of crucial importance in the description of particle yields in proton induced processes and in $e^{+} e^{-}$[ [12], as well as in peripheral heavy ion collisions [20].

In Fig. 11 the predictions of two different canonical statistical models for $\bar{B} / B$ ratios in pp collisions are included. The main difference between these models is contained in the implementation of baryon number and isospin conservation as well as how additional strangeness suppression is introduced.

(I) The calculation in this statistical model [ 24] is a full canonical one with fixed baryon number, strangeness and electric charge identical to those of initial state. Also, an extra strangeness suppression is needed to reproduce the experimental multiplicities. This is done by considering the number of newly produced $\langle s \bar{s}\rangle$ pairs as an additional charge to be found in the final hadrons. The $s \bar{s}$ pairs fluctuate according to a Poisson distribution and its mean number is considered a free parameter to be fitted [ 24]. The parameters used for the prediction of $\Omega^{+} / \Omega^{-}$ratio $(T$, global volume $V$ sum of single cluster volumes and $\langle s \bar{s}\rangle$ ) have been obtained by a fit to preliminary NA49 pp data [ 25] yielding $T=$ $183.7 \pm 6.7 \mathrm{MeV}, V T^{3}=6.49 \pm 1.33$ and $\langle s \bar{s}\rangle=0.405 \pm 0.026$ with a $\chi^{2} /$ dof $=11.7 / 9$. It must be pointed out that $\Omega^{+} / \Omega^{-}$ratio is actually independent of the $\langle s \bar{s}\rangle$ parameter and only depends on $T$ and $V$ (see also Fig.4).

(II) Here we first approximate the conservation of baryon number and electric charge by the GC ensemble. Under thermal conditions at top SPS energies this approximation leads to deviations from the exact $\mathrm{C}$ results in pp collisions by at most 20-30\% [ 22]. Strangeness conservation is, however, implemented on the canonical level following the procedure proposed in [20]. It accounts for strong correlations of produced strange particles due to constraints imposed by the locality of the conservation laws. In pp collisions strangeness is not distributed in the whole volume of the fireball but is strongly correlated. A correlation volume parameter $V_{0}=4 \pi R_{0}^{3} / 3$ is introduced, where $R_{0} \sim 1 \mathrm{fm}$ is a typical scale of QCD interactions. Previous analysis of WA97 pA data yields: $R_{0} \sim 1.12 \mathrm{fm}$ corresponding to $V_{0} \simeq 5.8 \mathrm{fm}^{3}$. Note that hidden strange particles are not canonically suppressed in this approach. Analysis of experimental data in AA collisions has shown that $T$ and $\mu_{B}$ are almost entirely determined by the collision energy and only dependent weakly on the number of participants [ [6]. $4 \pi$ results of NA49 on $\bar{p} / \pi$ and $\pi / A_{\text {part }}$ ratios in pp and $\mathrm{PbPb}$ collisions coincides within 20-30\%. In terms of the SM this can be understood if $T$ and $\mu_{B}$ in pp and $\mathrm{PbPb}$ collisions have similar values. We take $T \simeq 158 \mathrm{MeV}$ and $\mu_{B} \simeq 238 \mathrm{MeV}$ as obtained from SM analysis of full phase-space NA49 $\mathrm{Pb}-\mathrm{Pb}$ data [ 12]. The volume of the fireball $V \sim 17 \mathrm{fm}^{3}$ and the charge chemical potential in pp was then found to reproduce the average charge and baryon number in the initial state.

The predictions of the statistical models are shown in fig 1 (right). In these approaches the $\bar{B} / B$ ratio increases linearly with the strangeness content of the baryon. For comparison, both figures include preliminary data on the $\bar{B} / B$ ratio at midrapidity by NA49 [25] (stars). Roughly $45 \Omega^{-}$have been so far extracted, no $\bar{\Omega}$ has been observed. With a $95 \%$ confidence 


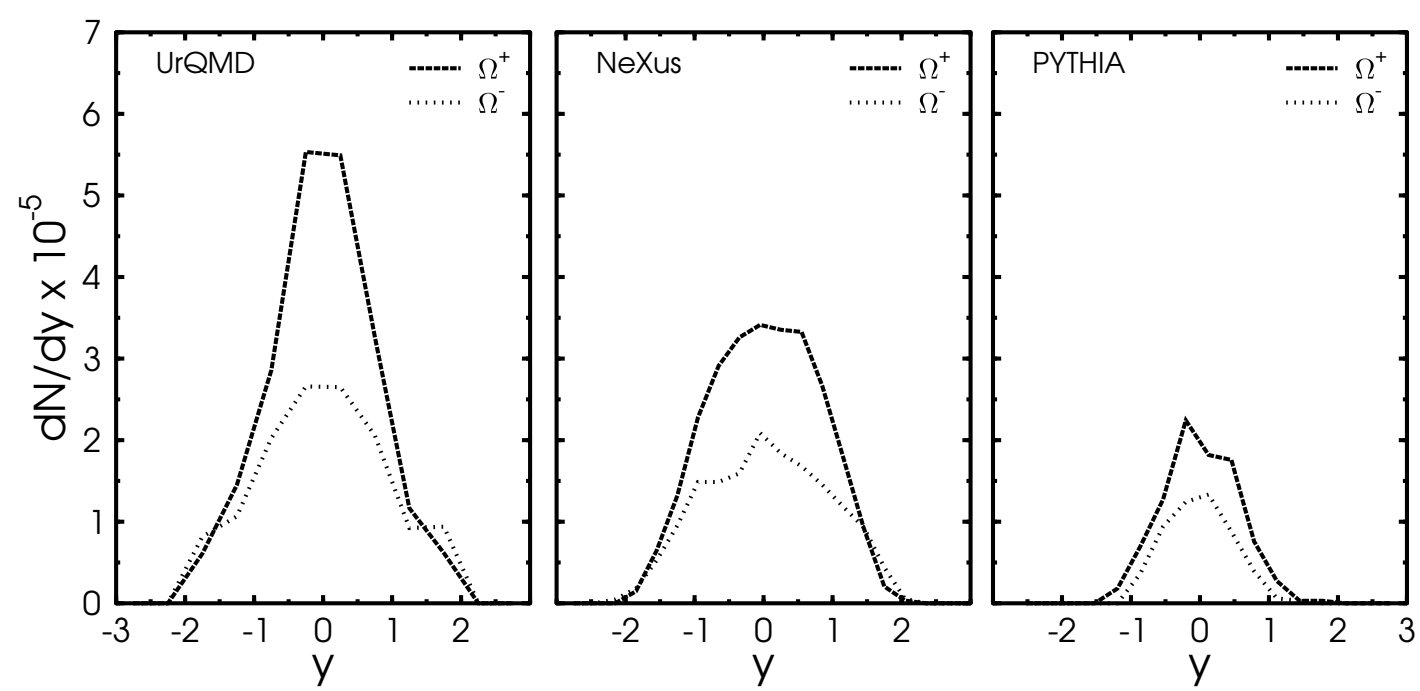

Fig. 2. Rapidity density of anti-Omegas and Omegas in pp interactions at $160 \mathrm{GeV}$ as predicted by UrQMD, NeXus, PYTHIA.

level, the $\bar{\Omega} / \Omega$ ratio in this measurement is below 0.5 [23]. However, it must be stressed that in the statistical models one can calculate particle production only in full phase space and all quoted predictions refer to fully integrated particle spectra.

Large deviations of statistical models from the data seen in Fig.1b are to be expected as at midrapidity $\bar{B} / B$ ratios are known to be much larger than in the whole phase space. To make predictions for particle ratios at midrapidity one can use the canonical model II. Following the procedure described in [20] we first choose $T \simeq 168 \mathrm{MeV}$, from the fit to the midrapidity WA97 $\mathrm{PbPb}$ data [7], and $\mu_{B} \simeq 130 \mathrm{MeV}$ to reproduce $\bar{p} / p \simeq 0.22$ in pp collisions. With these parameters the agreement of the model II with NA49 data is seen in Fig.1 to be quite satisfactory.

\section{Rapidity-, mass- and volume dependencies}

The rapidity dependence of $\Omega$ and $\bar{\Omega}$ yield is studied in Fig. 2 within different string models. The results were calculated in pp interactions at $160 \mathrm{GeV}$ within PYTHIA, NeXuS and UrQMD (from top to bottom). As can be seen, the $\bar{\Omega} / \Omega$ ratio is largest around mid-rapidity.

The $\bar{\Omega} / \Omega$ ratio is fairly robust - different string-model implementations (PYTHIA, UrQMD, NeXuS) all agree in their predictions within $\pm 20 \%$. The value of $\bar{\Omega} / \Omega>1$ in $\mathrm{p}+\mathrm{p}$ reactions at the SPS is a generic feature of the string-fragmentation. However, as shown in Table I the total $4 \pi$-yields of $\Omega$ 's and $\bar{\Omega}$ 's may differ by a factor of four among the different string models. The statistical models are in general giving more consistent results, however deviations up to $20 \%$ are not excluded.

In string models, the particle abundances depend on the parameters chosen for the fragmentation scheme, while in statistical models they reflect the differences between the 


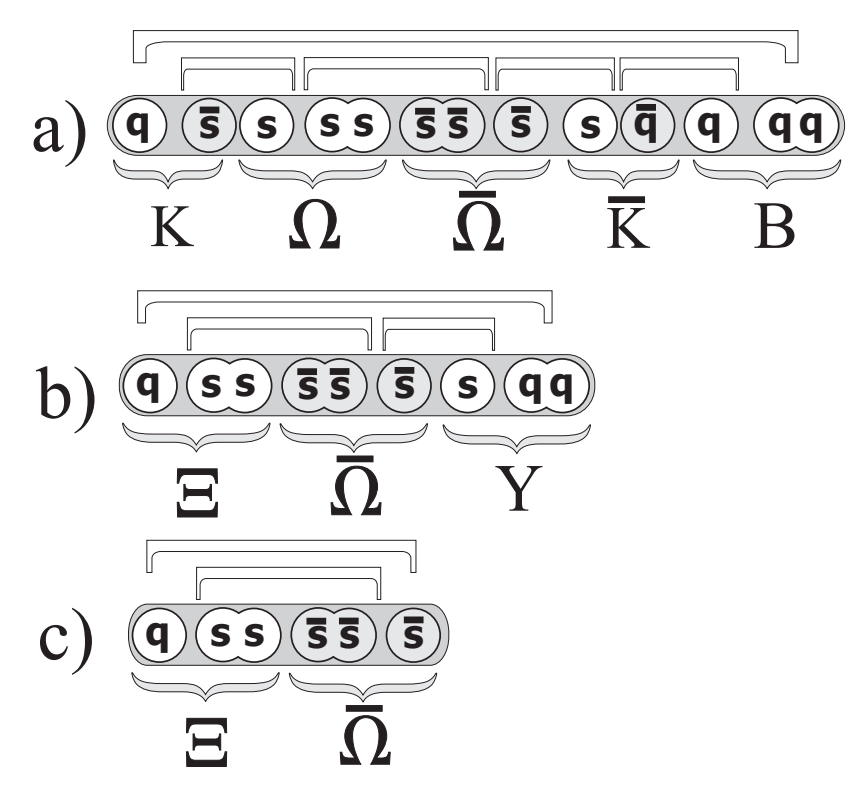

Fig. 3. Fragmentation of a color field into quarks and hadrons. While in large strings $\Omega \mathrm{s}$ and $\bar{\Omega}$ se produced in equal abundance (top), small strings suppress $\Omega$ s at the string ends.

ensembles chosen. Thus, the absolute yields allow to distinguish between the implementations once experimental data becomes available.

In order to understand the large $\bar{\Omega} / \Omega$ values predicted by string models one elucidates in Fig. 3 the color flux tube break-up mechanism. Fig. 3 shows the fragmentation of the color field into quark-antiquark pairs, which then coalesce into hadrons. While in large strings $\Omega$ s and $\bar{\Omega}$ s are produced in equal abundance (a), low-mass strings in UrQMD suppress $\Omega$ production at the string ends (b), while in $\mathrm{NeXuS} \bar{\Omega}$ 's are enhanced (c). Thus, the microscopic method of hadronization leads to a strong imbalance in $\bar{\Omega} / \Omega$ ratio in lowmass strings.

The $\bar{\Omega} / \Omega$ ratio depends in a strongly non-linear fashion on the mass of the fragmenting string. Fig. $\overline{4}$ shows the $\bar{\Omega} / \Omega$ ratio as a function of the mass of the fragmenting string (i.e. different beam energies in pp). One clearly observes a strong enhancement of $\bar{\Omega}$ production at low energies, while for large string masses the ratio approaches the value of $\bar{\Omega} / \Omega=1$ (which should be reached in the limit of an infinitely long color flux tube).

However, it should be noted that recently a new class of string models utilizing partonbased Gribov-Regge theory has been proposed which are capable of generating an $\bar{B} / B$ ratio of less than one [26].

Statistical models, on the other hand, are not able to yield a ratio of $\bar{\Omega} / \Omega>1$. This can be easily understood in the GC formalism, where $\bar{B} / B$ ratio is very sensitive to the baryon-chemical potential $\mu_{B}$. For finite baryon-densities, the $\bar{B} / B$ ratio will always be $<1$ and only in the limit of $\mu_{B}=0$ may $\bar{\Omega} / \Omega=1$ be approached. These features survive in the 


\begin{tabular}{lrr} 
Model & $\Omega\left(\times 10^{-4}\right)$ & $\bar{\Omega}\left(\times 10^{-4}\right)$ \\
\hline NeXus & 0.48 & 0.79 \\
PYTHIA & 0.17 & 0.30 \\
UrQMD & 0.66 & 1.05 \\
Canonical Model I & 0.46 & 0.31 \\
Canonical Model II & 0.41 & 0.24
\end{tabular}

Table 1. $4 \pi$ particles yields in pp collisions at $160 \mathrm{GeV}$

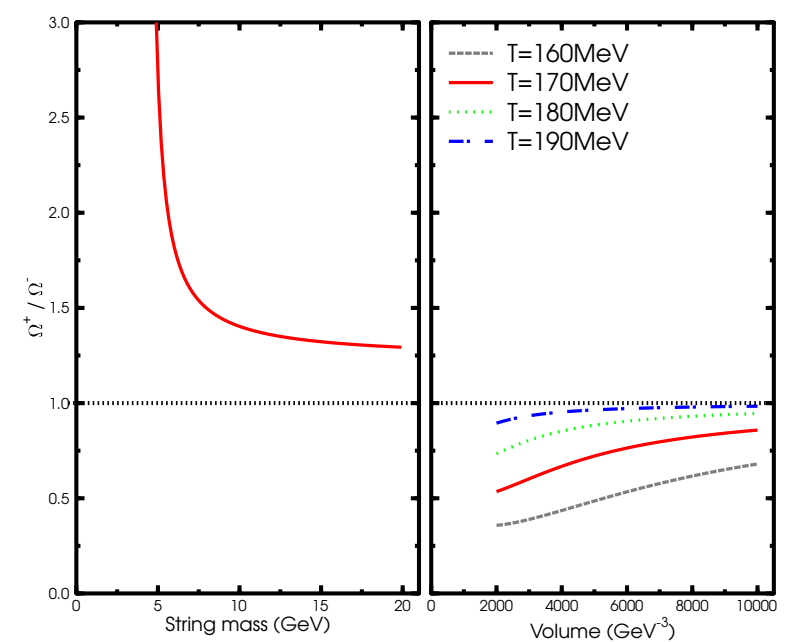

Fig. 4. Left: $\bar{\Omega} / \Omega$ ratio as a function of string mass. Right: $\bar{\Omega} / \Omega$ ratio as a function of the volume in Modell II.

canonical framework, where the GC fugacities are replaced by ratios of partition functions [12]. This is shown in Fig. 4 (right) where the ratio $\bar{\Omega} / \Omega$ in pp collisions (according to the previously described model I) is plotted as a function of volume for four different temperatures. Hence, finite size corrections in the statistical model actually lead to the opposite behavior[21] in the ratio of $\bar{\Omega} / \Omega$ vs. system-size (i.e. volume replacing stringmass) than that observed in the fragmenting color flux tube picture.

\section{Balance Functions}

While the $\bar{\Omega} / \Omega$ ratio provides us with important information on the dynamics of hadronization, it does not yield any information on the time-scales at which hadronization occurs: Balance functions offer a unique model-independent formalism to probe the time-scales of a deconfined phase and subsequent hadronization [27]. Late-stage production of quarks could be attributed to three mechanisms: formation of hadrons from gluons, conversion of the non-perturbative vacuum energy into particles, or hadronization of a quark gas at 
constant temperature. Hadronization of a quark gas should approximately conserve the net number of particles due to the constraint of entropy conservation. Since hadrons are formed of two or more quarks, creation of quark-antiquark pairs should accompany hadronization. All three mechanisms for late-stage quark production involve a change in the degrees of freedom. Therefore, any signal that pinpoints the time where quarks first appear in a collision would provide valuable insight into understanding whether a novel state of matter has been formed and persisted for a substantial time.

The link between balance functions and the time at which quarks are created has a simple physical explanation. Charge-anticharge pairs are created at the same location in space-time, and are correlated in rapidity due to the strong collective expansion inherent to a relativistic heavy ion collision. Pairs created earlier can separate further in rapidity due to the higher initial temperature and due to the diffusive interactions with other particles. The balance function, which describes the momentum of the accompanying antiparticle, quantifies this correlation. The balance function describes the conditional probability that a particle in the bin $p_{1}$ will be accompanied by a particle of opposite charge in the bin $p_{2}$ :

$$
B\left(p_{2} \mid p_{1}\right) \equiv \frac{1}{2}\left\{\rho\left(b, p_{2} \mid a, p_{1}\right)-\rho\left(b, p_{2} \mid b, p_{1}\right)+\rho\left(a, p_{2} \mid b, p_{1}\right)-\rho\left(a, p_{2} \mid a, p_{1}\right)\right\},
$$

where $\rho\left(b, p_{2} \mid a, p_{1}\right)$ is the conditional probability of observing a particle of type $b$ in bin $p_{2}$ given the existence of a particle of type $a$ in bin $p_{1}$. The label $a$ might refer to all negative kaons with $b$ referring to all positive kaons, or $a$ might refer to all hadrons with a strange quark while $b$ refers to all hadrons with an antistrange quark. Balance functions will be discussed in greater detail in other articles of these proceedings [28, 29, 30].

\section{Conclusions}

The $\bar{\Omega} / \Omega$ has been found to be extremely sensitive on the dynamics of hadronization in $\mathrm{p}+\mathrm{p}$ reactions. Within the fragmenting color flux tube models we have predicted that $\bar{\Omega} / \Omega$ ratio is significantly above unity. This is in strong contrast to statistical model results which always imply that $\bar{B} / B$ ratios are bellow or equal to unity in proton+proton reactions. Since this observable is accessible by NA49 measurements at the SPS it can provide an excellent test to distinguish the statistical model hadronization scenario from that of microscopic color-flux tube dynamics - first very preliminary results seem to support the statistical hadronization hypothesis. Balance functions yield complimentary information and offer a unique, model-independent formalism to probe the time-scale of hadronization in heavy nucleus-nucleus collisions.

\section{Acknowledgement(s)}

We thank K. Kadjia (NA49) for fruitful and stimulating discussions. (S.A.B.) acknowledges support from RIKEN, Brookhaven National Laboratory and DOE grants DE-FG0296ER40945 as well as DE-AC02-98CH10886. (K.R) acknowledges KBN grant, KBN2P03B 03018. 


\section{References}

1. S. A. Bass, M. Gyulassy, H. Stöcker and W. Greiner, J. Phys. G25, R1 (1999).

2. H. Stöcker, W. Greiner and W. Scheid, Z. Phys. A286, 121 (1978); D. Hahn and H. Stöcker, Nucl. Phys. A452, 723 (1986).

3. R. Stock, Phys. Lett. B456, 277 (1999).

4. J. Rafelski and B. Müller, Phys. Rev. Lett. 48, 1066 (1982); P. Koch, B. Müller and J. Rafelski, Phys. Rep. 142, 167 (1986).

5. J. Letessier, J. Rafelski and A. Tounsi, Phys. Lett. B321, 394 (1994); J. Rafelski and J. Letessier, Phys. Rev. Lett. 85, 4695 (2000).

6. J. Cleymans and K. Redlich, Phys. Rev. Lett. 81, 5284 (1998); F. Becattini, et al., Phys. Rev. C 64, 024901 (2001).

7. P. Braun-Munzinger, I. Heppe and J. Stachel, Phys. Lett. B465, 15 (1999).

8. P. Braun-Munzinger, J. Stachel, J. P. Wessels and N. Xu, Phys. Lett. B344, 43 (1995); Phys. Lett. B365, 1 (1996); P. Braun-Munzinger and J. Stachel, Nucl. Phys. A606, 320 (1996); P. Braun-Munzinger, D. Magestro, K. Redlich and J. Stachel, Phys. Lett. B 518, 41 (2001).

9. C. Spieles, H. Stocker and C. Greiner, Eur. Phys. J. C 2 (1998) 351. C. Greiner and H. Stöcker., Phys. Rev. D44, 3517 (1992). S. A. Bass et al., Phys. Rev. Lett. 81, 4092 (1998).

10. M. Bleicher and J. Aichelin, Phys. Lett. B 530 (2002) 81 arXiv:hep-ph/0201123.

11. R. Hagedorn, Nuovo Cim. Suppl. 3, 147 (1965); R. Hagedorn and J. Ranft, Nuovo Cim. Suppl. 6, 169 (1968).

12. F. Becattini, Z. Phys. C69 (1996) 485; F. Becattini and U. Heinz, Z. Phys. C76, 269 (1997).

13. A. Casher, H. Neuberger and S. Nussinov, Phys. Rev. D 20, 179 (1979).

14. M. Bleicher et al., Phys. Rev. Lett. in print and arXiv:hep-ph/0111187.

15. J. S. Schwinger, Phys. Rev. 82 (1951) 664.

16. M. Bleicher et al., J. Phys. G 25 (1999) 1859, S. A. Bass et al., Prog. Part. Nucl. Phys. 41 (1998) 225.

17. H.J. Drescher et al. Phys. Rep. 350 (2001) 93.

18. H.-U. Bengtsson and T. Sjöstrand, Comput. Phys. Commun. 46 (1987) 43.

19. R. Hagedorn, CERN Rep. 71 (1971); E. Shuryak, Phys. Lett. B42 (1972) 357; J. Rafelski, Phys. Lett. B97 (1980) 279; K. Redlich and L. Turko, Z. Phys. C5, (1980) 201. C.M. Ko, V. Koch, Z. Lin, K. Redlich, M. Stephanov and X.N. Wang, Phys. Rev. Lett. 86 (2001) 5438, and references therein; P. Braun-Munzinger, et al. hep-ph/0106066.

20. J. S. Hamieh, K. Redlich and A. Tounsi, Phys. Lett. B486 (2000) 61; J. Phys. G27, 413 (2001).

21. A. Keränen and F. Becattini, Phys. Rev. C65 (2002) 044901.

22. M. Gorenstein and M. Gazdzicki Phys. Lett. B483, 60 (2000).

23. NA49 Collaboration, Addendum-10 to Proposal CERN/SPSC/P264.

24. F. Becattini, G. Passaleva, hep-ph/0110312

25. J. Bachler et al., Nucl. Phys. A661 (1999) 45; R. A. Barton et al., J. Phys G27 (2001) 
367; V. Afanasev et al., Phys. Lett. B491 (2000) 59;

26. F. M. Liu, J. Aichelin, M. Bleicher, H. J. Drescher, S. Ostapchenko, T. Pierog and K. Werner, arXiv:hep-ph/0202008.

27. S. A. Bass, P. Danielewicz and S. Pratt, Phys. Rev. Lett. 85, 2689 (2000).

28. S. Pratt, these proceedings.

29. G. Westfall et al., these proceedings.

30. M. Tonjes et al., these proceedings. 